Ingeniería Investigación y Tecnología, ISSN 2594-0732,

IX. 3. 217-229, 2008 (artículo arbitrado)

DOI: http://dx.doi.org/10.22201/fi.25940732e.2008.09n3.017

\title{
Reducción del retardo en el cambio de canal EN SERVICIOS IPTV
}

\section{REdUCTION OF CHANNEl ZAPPING dElAY IN IPTV SERVICES}

\author{
F. Moumtadi, M. Escobar-Argota, R. López-Moreno y S. Landeros-Ayala \\ Facultad de Ingeniería, UNAM, México \\ E-mails: fatimoum@hotmail.com,marlene_escobar_a@yahoo.com.mx, \\ ricardo_lopez_m@yahoo.com.mxysland@fi-b.unam.mx
}

(Recibido: diciembre de 2006; aceptado: enero de 2008)

\begin{abstract}
Resumen
En el artículo se analiza el retraso que se presenta en un sistema de IPTV cuando un usuario cambia de canal y se considera un nuevo algoritmo para reducirlo. Basado en el método de grupos adyacentes, se reduce el tiempo de adquisición del nuevo canal haciendo el pedido no solamente de ese canal, sino también de los canales adyacentes a él, tomando como principio que existe un ancho de banda suficiente y un principio de multicast de la transmisión (Chunglae, 2004). En la parte final del mismo, se comparan los resultados obtenidos contra los actuales y se identifican las posibilidades del algoritmo examinado.
\end{abstract}

Descriptores: IPTV, método de Grupos Adyacentes, retardo de cambio de canal, HG, IGMP.

\begin{abstract}
This paper analyses the existing delay within an IPTV system when a user selects another channel, and a new algorithm is used to reduce it. Based on the adjacent groups method, which reduces the time acquisition of a new channel placing the order not only for that channel but also for the adjacent channels. There are two principles under coonsideration, one that there exists a sufficient bandwidht, and the other, a multicast transmission (Chunglae, 2004). At the end of the paper, the obtained results are compared against the present ones and the possibilities of the algorithm are determined.
\end{abstract}

Keywords: IPTV, Adjacent Groups method, channel zapping delay, HG, IGMP.

\section{Introducción}

A lo largo de los últimos años, el incremento del ancho de banda en las redes de telecomunicaciones ha posibilitado la adopción, por parte de los proveedores de servicios, del sistema de televisión IPTV (Internet Protocol Television), que gestiona la información de sus programas en un esquema multicast posibilitando al proveedor a no transmitir su programación de manera ininterrumpida espe- rando que algún usuario se conecte al sistema, sino que el contenido únicamente llegue al usuario cuando éste lo solicite. Esta nueva modalidad exige un mayor ancho de banda disponible en el sistema, pero permite, en cambio, ofrecer de manera sencilla y eficiente servicios de televisión digital de siguiente generación sobre redes de banda ancha como: Oferta ilimitada de canales de televisión digital y música, programación de pago por evento, video por demanda, grabación 
personalizada de video, publicidad interactiva y servicios de información (Muñiz, 2005).

Estas ventajas se ven limitadas; por la necesidad de disminuir el retardo que se presenta cuando el usuario cambia el canal seleccionado, que puede variar de 2 a 3 seg (Full Service- VDSL, 2002), (Cisco Systems, 2006a), a fin de asegurar la calidad de servicio.

La televisión digital tradicional por cable, basada en receptores de tipo STB (Set Top Box), tiene la capacidad de recibir todos los canales del sistema televisivo en forma simultánea, lo que posibilita al usuario cambiar de canal sin retardos perceptibles. En contraposición, el sistema IPTV permite la gestión de flujos de canales individuales a la vez; la selección de otro canal genera un nuevo flujo de información, lo que provoca un retardo en la transmisión de la señal (Muñiz, 2005), (Siemens Communications and Juuniper Networks, 2006).

En la actualidad, se han presentado varias propuestas para disminuir el retardo como: la reducción en el tiempo de implementación del STB, optimización en el diseño de la red, decodificación y desencriptación de datos, los cuales representan el mayor porcentaje del retardo (Cisco Systems, 2006).
En este artículo se trabaja sólo en el retardo de la red, el cual se presenta únicamente para IPTV, a través de la implementación del método de grupos adyacentes.

\section{Arquitectura de IPTV y proceso de cambio del canal}

La figura 1 muestra la arquitectura de red y los componentes del sistema IPTV. Esta topología considera un esquema jerárquico. En la parte receptora, la puerta de enlace del usuario HG (Home Gateway) contiene el flujo de todos los STB conectados a él; el Multiplexor de acceso a la línea de abonado digital DSLAM (Digital Subscriber Line Access Multiplexer) concentra, a su vez, el flujo de todos los HG a él directamente conectados y, por último, el LHR (Last Hop Router) concentra el flujo de todos los DSLAM's. En la parte transmisora, el FHR (First Hop Router) contiene el flujo de todos los canales del sistema IPTV, mientras que la cabecera del sistema (IPTV headend) es la encargada de distribuir, almacenar y administrar todos los contenidos multicast del sistema IPTV (Chunglae, 2004), (Full Service-VDSL, 2002) y (Cisco Systems, 2006a).

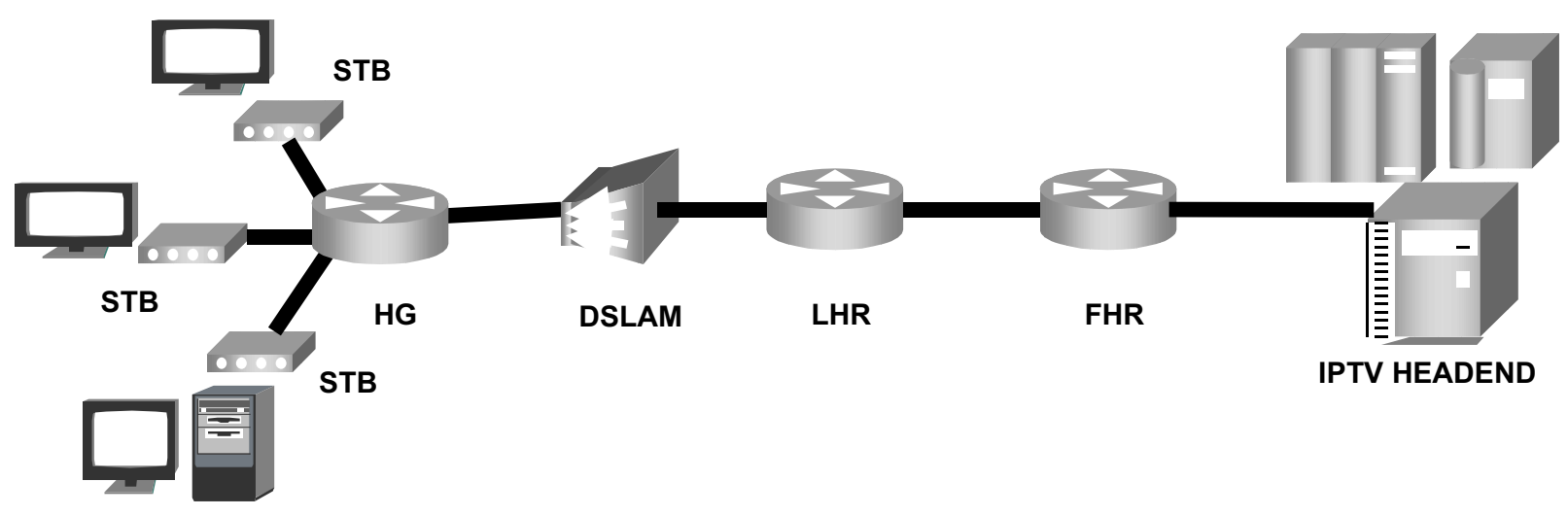

Figura 1. Arquitectura general del sistema IPTV 
F. Moumtadi, M. Escobar-Argota, R. López-Moreno y S. Landeros-ayala

Para la unión a un flujo multicast, el STB usa el protocolo de gestión de grupos en Internet IGMP (Internet Group Management Protocol) (Fenner, 1997), que se encarga de enviar mensajes de entrada y abandono de grupo (Join-Leave messages) al ruteador que le gestiona, el HG.

El HG responde a los mensajes de solicitud de membresía (Membership query messages) que el DSLAM le solicita para verificar su estado por medio de los mensajes de reporte de membresía (Membership report messages). El HG también utiliza IGMP, actuando como si fuera él mismo un host. De esta manera si el canal solicitado en el mensaje de entrada al grupo ya está disponible en el HG, éste simplemente copiará el flujo multicast y lo transmitirá inmediatamente al STB; si no, el proceso anterior se repite para los ruteadores siguientes, el DSLAM y el LHR. El LHR debe soportar tanto el IGMP para comunicarse con el DSLAM como el Protocolo Multicast Independiente de Baja Densidad (Protocol Independent Multicast Sparse-Mode) PIM-SM (Fenner et al., 2003) para comunicarse con el FHR. El LHR también envía mensajes PIMSM al FHR para notificarle que el estado de membresía a grupos multicast ha cambiado, logrando de esta manera que el flujo multicast correspondiente a un canal se detenga o se retransmita un nuevo flujo.

\section{Método de grupos adyacentes}

El retardo en sistemas IPTV debe de entenderse como "el tiempo transcurrido entre el envío del primer mensaje de abandono de grupo hasta la recepción del primer paquete de flujo multicast por parte del STB" (Chunglae, 2004), (Full Service-
VDSL, 2002) y (Cisco Systems, 2006a) y una de las propuestas actuales para reducir su magnitud es la aplicación del método de grupos adyacentes: asumiendo que el ancho de banda de la red de acceso (esto es, entre el LHR y el HG) es lo suficientemente grande como para soportar diversos flujos multicast de manera simultánea, ante la solicitud por parte del STB de adquirir un nuevo canal mediante el envío de un mensaje de entrada al grupo, el HG podría no sólo enviar el mensaje de entrada correspondiente al grupo de ese canal, sino también para los grupos de los canales adyacentes (Figura 2).

De esta manera, los flujos multicast pertenecientes a los canales adyacentes del canal solicitado previamente por el STB estarán siempre disponibles en el HG; cuando el STB solicite la adquisición de un canal adyacente, el HG podrá enviar el tráfico multicast respectivo inmediatamente, lo que permite reducir considerablemente el retardo en el cambio de canal (Chunglae, 2004).

\section{Simulación de la implementación del método de grupos adyacentes}

La simulación fue desarrollada en el software Matlab y se realizó en base al sistema mostrado en la figura 1. Los elementos en conjunto representan sólo una sección de un sistema para IPTV que da servicio a una gran cantidad de suscriptores. Cuando un STB cambie de canal se medirá el retardo en la adquisición de canal (Join latency) de acuerdo a la disponibilidad del canal deseado en los ruteadores; es decir, si el canal solicitado se encuentra disponible en el HG, el tiempo de adquisición será

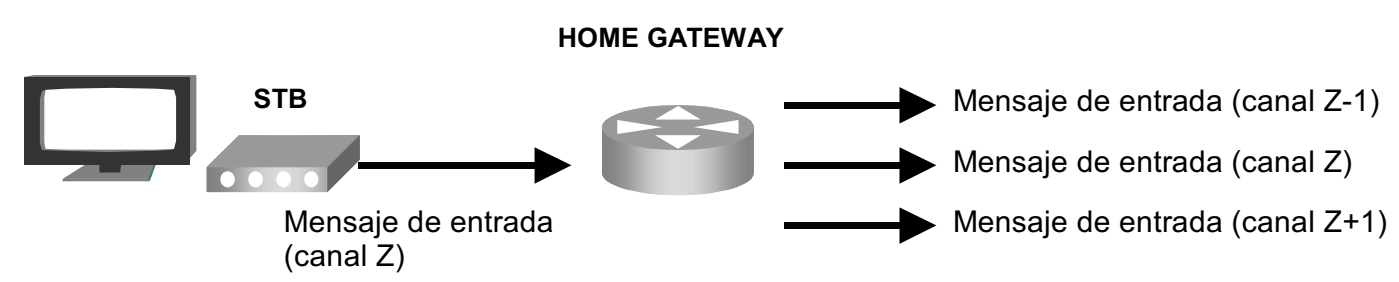

Figura 2. Concepto del Método de Grupos Adyacentes 
de 50 ms; si el mensaje de entrada al grupo tiene que viajar hasta el DSLAM, el tiempo será de 150 ms y si es hasta el LHR o FHR, será de 500 ms o $600 \mathrm{~ms}$, respectivamente (Full Service-VDSL, 2002) y (Cisco Systems, 2006b), (Figura 3).

El método de grupos adyacentes fue aplicado con dos canales adyacentes, uno hacia arriba y otro hacia abajo, y se analizó su influencia en el tiempo de cambio de canal que experimentan los usuarios.

Para la simulación se tomó en cuenta que se realizan diez cambios de canal entre los tres STB's del sistema. Se eligió un STB al azar para cada cambio. Los cambios de canal se efectuaron de dos formas distintas, cambios hacia canales aleatorios y cambios hacia canales adyacentes.

Se analizaron cuatro escenarios diferentes:

1. Metodología de cambio tradicional con cambios hacia canales adyacentes;

2. Metodología de cambio mejorada con cambios hacia canales adyacentes;

3. Metodología de cambio tradicional con cambios hacia canales aleatorios;

4. Metodología de cambio mejorada con cambios hacia canales aleatorios.
Para representar la cantidad total de suscriptores del sistema IPTV se añadieron más usuarios al DSLAM, mismos que se cargaron al LHR más un flujo del $75 \%$ total de los canales en el LHR para mantener una configuración PIM-SM.

Sobre cada escenario se hicieron variar tanto el número de usuarios como el de canales para analizar la influencia de estos en el tiempo de cambio de canal.

\section{Análisis de resultados}

Los resultados se presentan como el promedio de los diez cambios de canal para diez iteraciones del programa.

La figura 4 ilustra los resultados obtenidos para los escenarios 1 y 2 tomando en cuenta 100 canales y 50 usuarios. Se observa que al aplicar el método de grupos adyacentes, existe una reducción considerable del tiempo de adquisición de canal cuando el usuario cambia a canales adyacentes. Con el método tradicional se obtiene un promedio de las 10 iteraciones de alrededor de $380 \mathrm{~ms}$. El tiempo de retardo con el método de grupos adyacentes se mantiene constante y con un valor de $50 \mathrm{~ms}$, debido a que los canales que son solicitados por los STB's están siempre disponibles dentro del HG; por lo tanto, el STB adquirirá el canal de forma instantánea. La reducción que se alcanzó con el método fue de 330 ms aproximadamente.

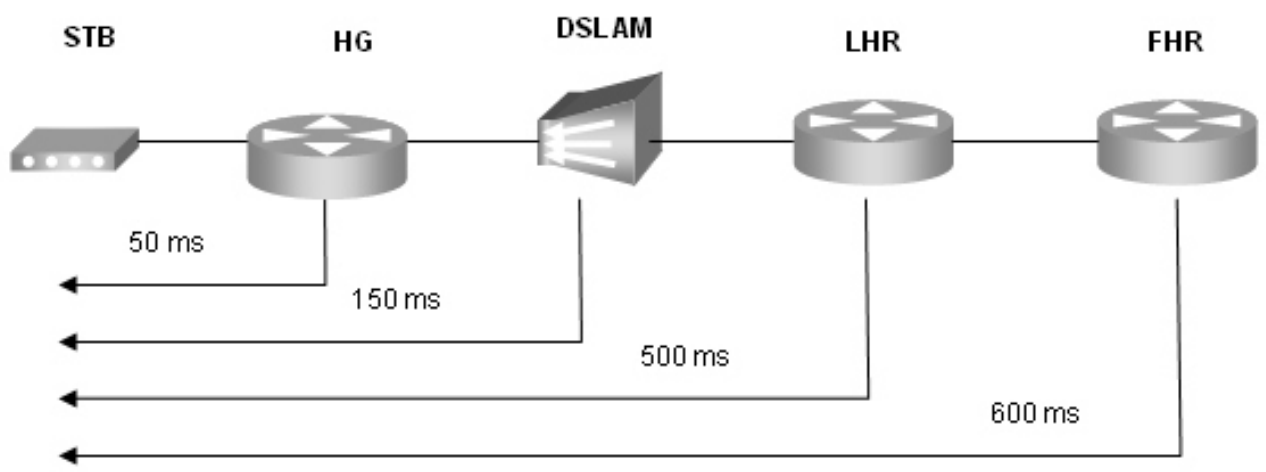

Figura 3. Diagrama de Simulación 
F. Moumtadi, M. Escobar-Argota, R. López-Moreno y S. Landeros-ayala

La figura 5 ilustra los resultados para los escenarios 3 y 4 para los mismos 100 canales y 50 usuarios. Se observa que el retardo se mantiene en un promedio de $380 \mathrm{~ms}$; esta vez, con el método implementado se obtiene un retardo con un valor promedio aproximado de 200 ms, logrando una reducción del tiempo de adquisición cerca de

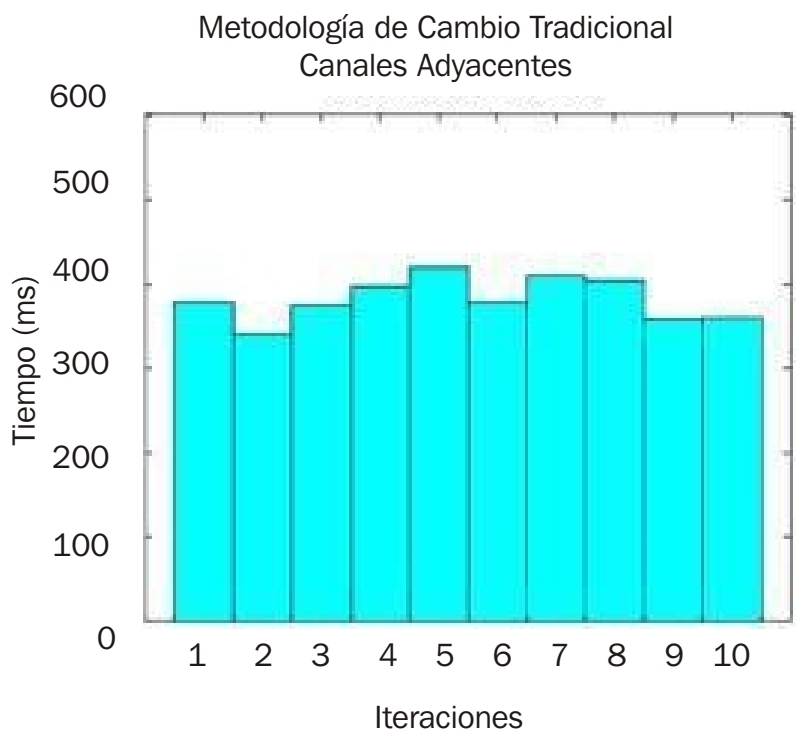

a.
180 ms. Esta reducción del tiempo no es tan amplia como cuando se cambia a canales adyacentes, debido a que, con la disponibilidad de los canales adyacentes en el HG, el DSLAM o el LHR, los canales solicitados pueden o no estar disponibles en estos ruteadores.

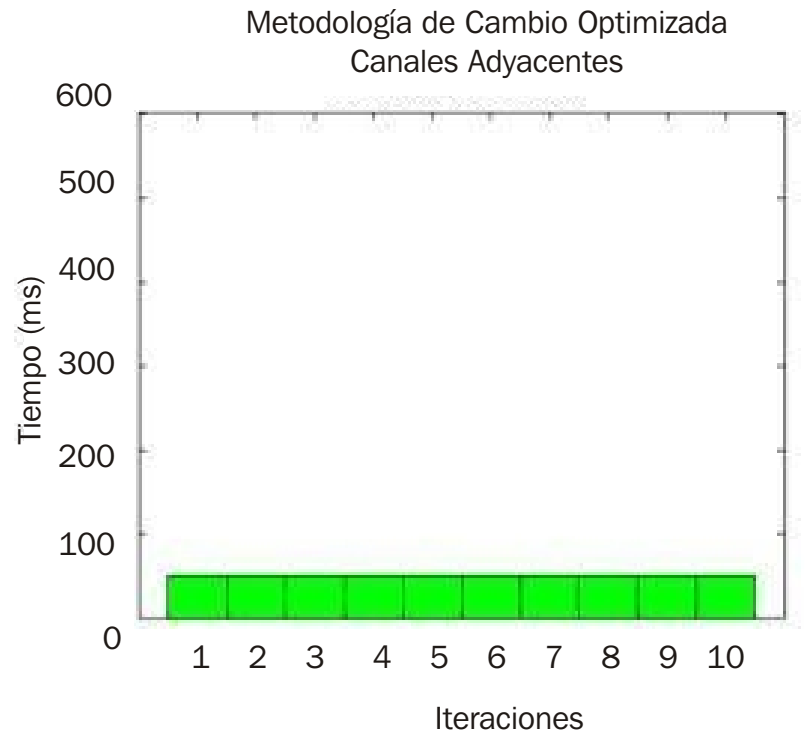

b.

Figura 4. Resultados para 100 canales y 50 usuarios; a) Escenario 1; b) Escenario 2

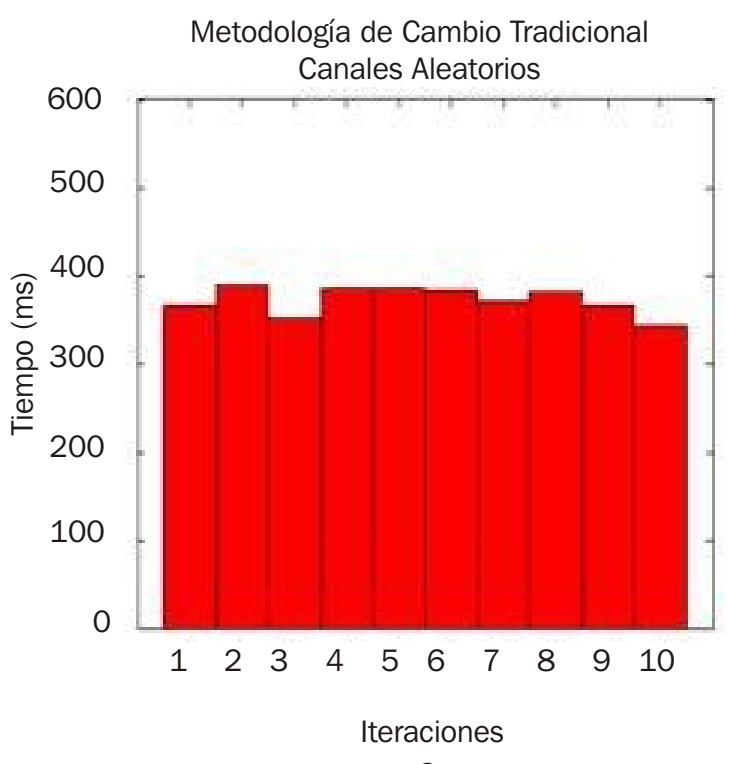

a.

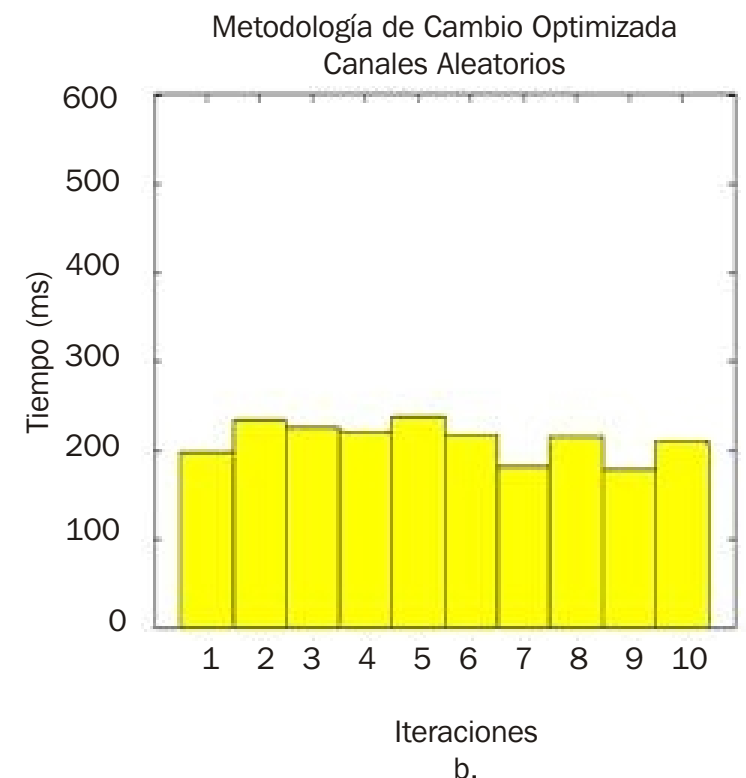

b.

Figura 5. Resultados para 100 canales y 50 usuarios; a) Escenario 3; b) Escenario 4 
Reducción del Retardo en el cambio de CANAL EN SERVICIOS IPTV

Tabla 1. Resultados de la simulación de los 4 escenarios

\begin{tabular}{cccccc}
\hline Usuarios & Canales & Canales & $\begin{array}{c}\text { Retardo con Método } \\
\text { Tradicional [ms] }\end{array}$ & $\begin{array}{c}\text { Rerardo con Método } \\
\text { Mejorado [ms] }\end{array}$ & Reducción [ms] \\
\hline \multirow{2}{*}{50} & \multirow{2}{*}{100} & Adyacentes & 380 & 50 & 330 \\
& \multirow{2}{*}{50} & Aleatorios & 380 & 200 & 180 \\
& \multirow{2}{*}{50} & Adyacentes & 450 & 50 & 400 \\
& \multirow{2}{*}{50} & Aleatorios & 450 & 300 & 150 \\
& \multirow{2}{*}{50} & Adyacentes & 280 & 50 & 230 \\
& \multirow{2}{*}{100} & Aleatorios & 280 & 150 & 130 \\
& \multirow{2}{*}{100} & Adyacentes & 300 & 50 & 250 \\
& \multirow{2}{*}{100} & Aleatorios & 300 & 170 & 130 \\
& Adyacentes & 430 & 50 & 380 \\
& & Aleatorios & 430 & 320 & 110 \\
\hline
\end{tabular}

La tabla 1 presenta los resultados de la simulación al variar el número de canales y el número de usuarios.

Cuando aumentamos el número de canales se aprecia un crecimiento en el retardo (Tabla 1), debido a que es menos probable que el canal deseado esté siendo visto por algún otro usuario, por lo que el canal requerido se encontrará disponible más lejos del usuario que solicita el canal. Por el contrario, el retardo disminuye si decrece el número de canales, ya que es más probable que el canal deseado esté siendo visto por algún otro usuario, así el canal requerido se encontrará disponible más cerca del usuario que solicite el canal.

Ahora bien, si en vez de variar el número de canales variamos el número de usuarios, al aumentar éstos se tiene una disminución del tiempo de retardo, a causa de que será más factible que el canal deseado ya esté siendo visto por algún otro usuario del sistema, por lo que el canal se hallará disponible más cerca del STB que hace la petición de adquisición. El retardo aumenta si disminuimos el número de usuarios, ya que es menos probable que el canal requerido se halle más cerca del STB que lo demanda.
Para analizar mejor cómo varía el tiempo de adquisición de canal, respecto al número de canales y al número de usuarios con las dos metodologías de cambio de canal, se realizaron las gráficas de las figuras 6 y 7 cuando los usuarios cambian a canales adyacentes y a canales aleatorios.

La figura 6 muestra las curvas para 25, 50 y 100 usuarios haciendo un barrido de canales bajo las condiciones de los escenarios 1 y 2 . La diferencia entre los resultados obtenidos por ambas metodologías también se ilustra. Se aprecia que al aumentar el número de canales el retardo aumenta desde 50 ms hasta un valor cercano a los $500 \mathrm{~ms}^{1}$, disminuyendo al aumentar el número de usuarios para la metodología de cambio tradicional. Para la metodología de cambio mejorada el retardo se mantiene constante en $50 \mathrm{~ms}^{1}$. Para analizar el comportamiento de la reducción del tiempo de adquisición de canal empleando el método de reducción se obtuvo la curva de la diferencia entre los resultados adquiridos con el método tradicional y los adquiridos con el método mejorado para 25, 50 y 100 usuarios; en esta última gráfica se puede apreciar qué tanto beneficio otorga el método

1 Hay que recordar que estos valores se constituyen mediante el promedio de las 10 iteraciones hechas al programa. 
de grupos adyacentes, el cual se incrementa conforme aumenta el número de canales y conforme disminuye el número de usuarios.

La figura 7 muestra las curvas para 25, $50 \mathrm{y}$ 100 usuarios haciendo un barrido de canales, ahora para los escenarios 3 y 4 . La diferencia entre los resultados obtenidos por ambas metodologías también se ilustra. Se observa también que el retardo crece al aumentar los canales. Al aplicar el método de grupos adyacentes se observa una pequeña reducción del retardo. Se ilustra la diferencia entre los métodos tradicional y mejorado, en donde se puede apreciar el ahorro de tiempo

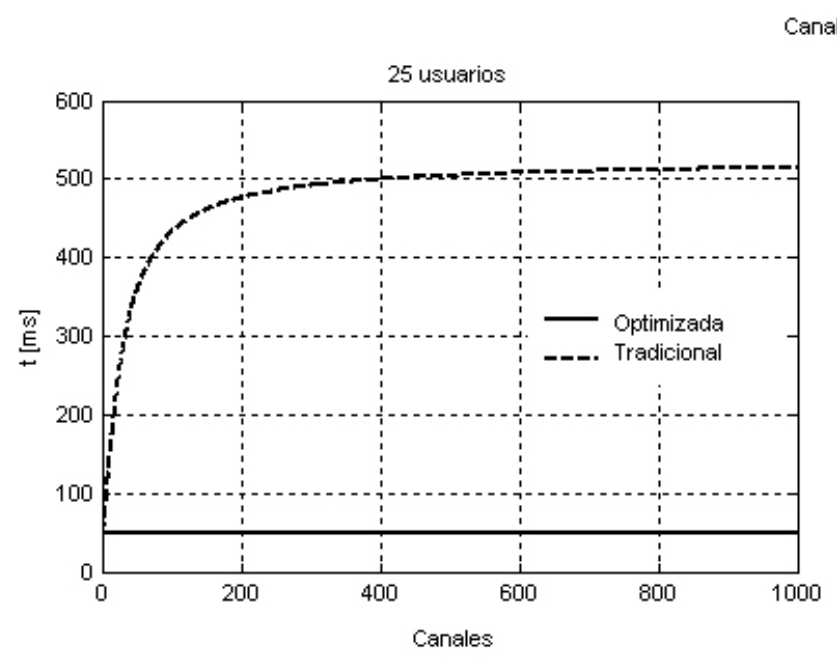

a)

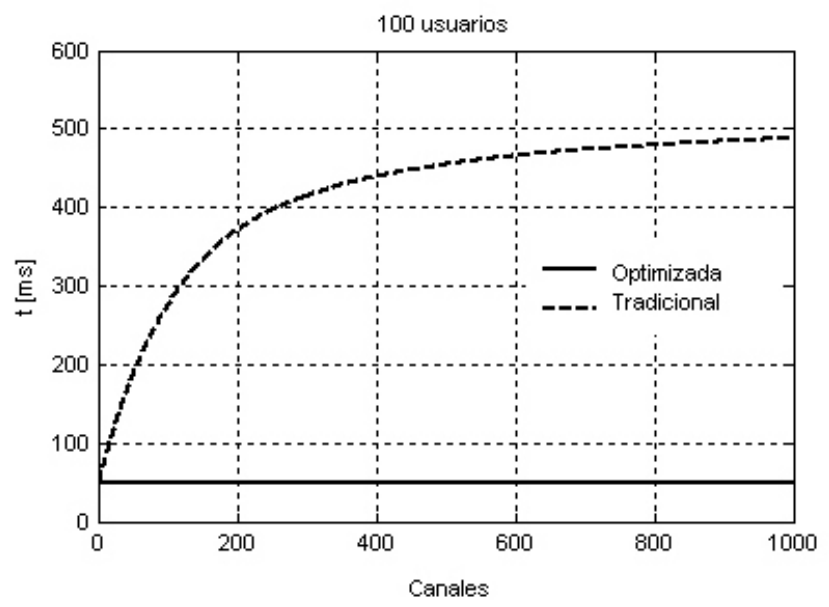

c)

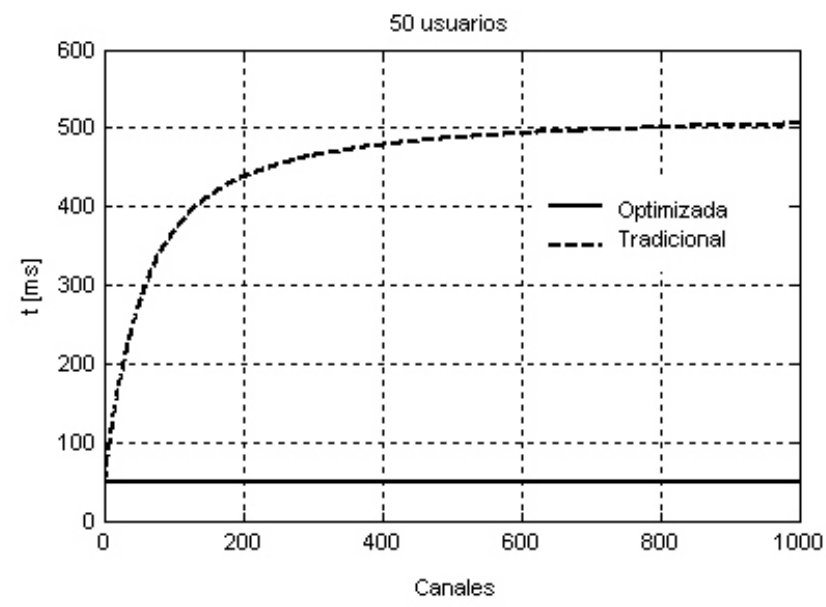

b)

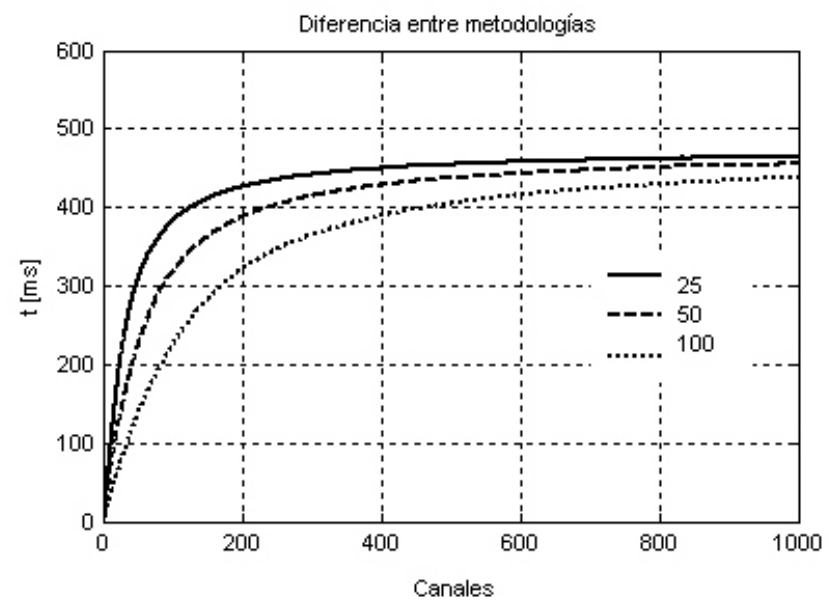

d)

Figura 6. Resultados obtenidos al variar el número de canales para cambios a canales adyacentes: a) 25 usuarios, b) 50 usuarios, c) 100 usuarios, d) Diferencia entre ambas tecnologías 
que se adquiere con el método de grupos adyacentes, con respecto al método tradicional de cambio de canal. Aquí el máximo beneficio no se logra conforme al aumento del número de canales, sino aproximadamente en el punto en el que el número de canales es igual al doble del número de usuarios, cuyo valor es cercano a 150 ms. Esto es porque con pocos canales se obtiene gran dispo- nibilidad en el DSLAM, tanto para el método tradicional como para el mejorado; conforme aumentan los canales la disponibilidad disminuye para los dos métodos, pero más con el tradicional, de ahí que la curva aumenta hasta llegar al máximo beneficio. A partir de este punto, la gráfica decrece debido a que la disponibilidad de canales disminuye, ahora más, con el método de grupos adyacentes.

Canales Aleatorios

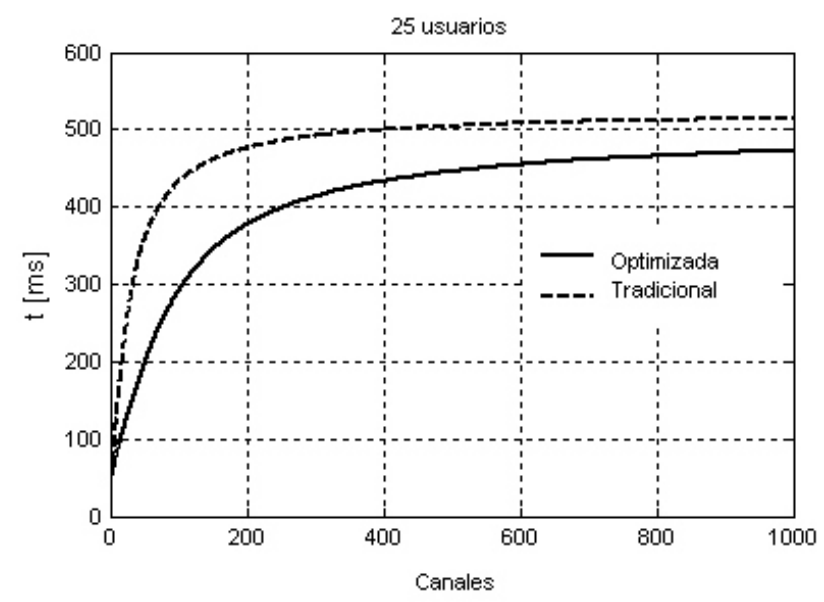

a)

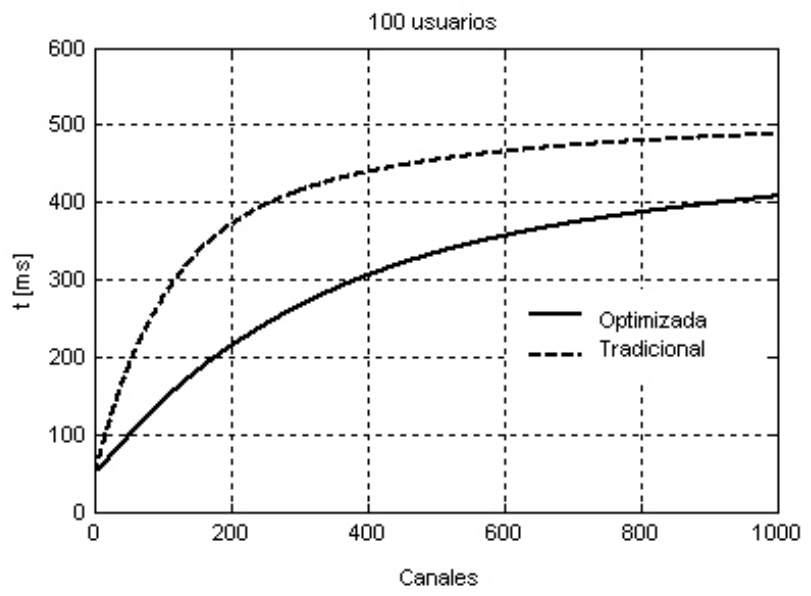

c)

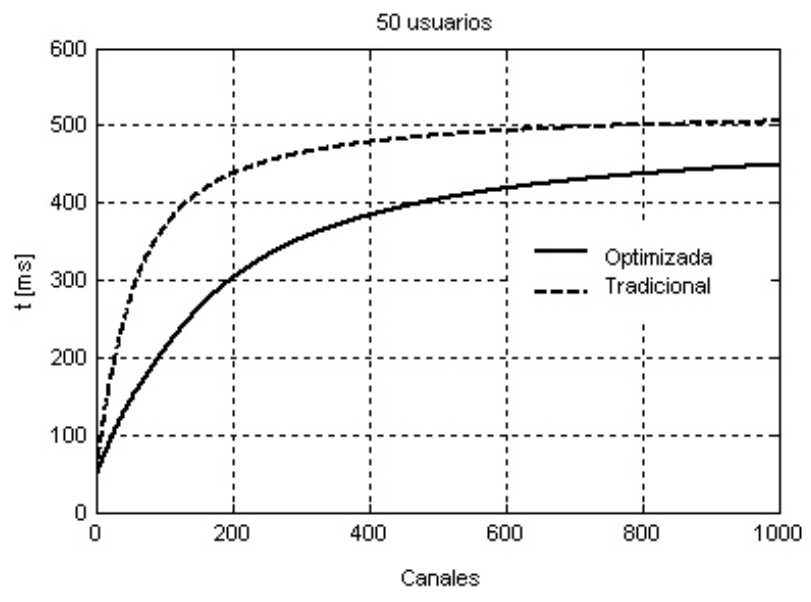

b)

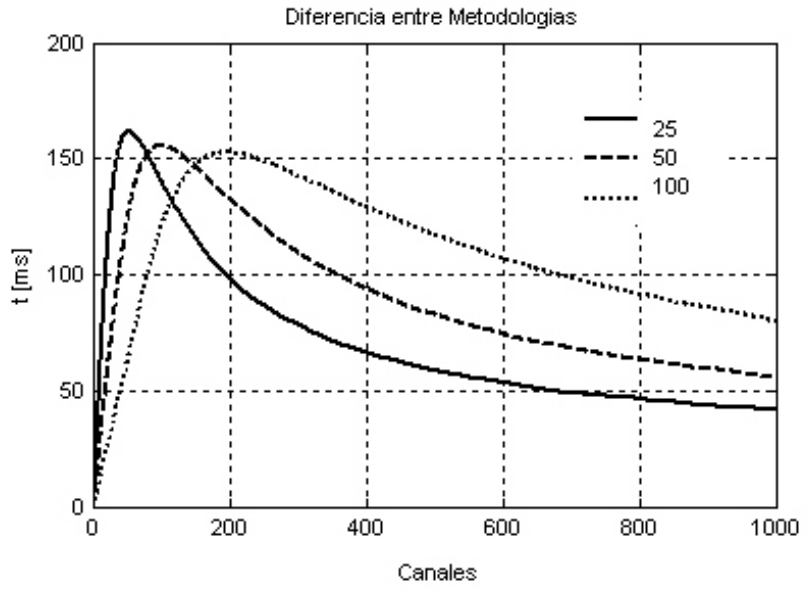

d)

Figura 7. Resultados obtenidos al variar el número de canales para cambios a canales aleatorios: a) 25 usuarios, a) 50 usuarios, a) 100 usuarios, a) Diferencia entre ambas tecnologías 
La figura 8 muestra las curvas para 50, 100 y 200 canales haciendo un barrido del número de usuarios bajo las condiciones de los escenarios 1 y 2. La diferencia entre los resultados obtenidos por ambas metodologías también se ilustra. Observamos, para canales adyacentes, que al aumentar el número de usuarios el retardo disminuye desde 500 ms hasta llegar al valor de $150 \mathrm{~ms}^{2}$ tanto para 50, 100 y 200 canales para la metodología de cambio tradicional. Para la metodología de cam- bio mejorada, el retardo se mantiene nuevamente constante en 50 [ms]. En la curva que muestra la diferencia entre los resultados obtenidos por los dos métodos, se observa el beneficio logrado al aplicar el método de grupos adyacentes, el cual decrece conforme aumenta el número de usuarios y conforme disminuye el número de canales.

2 A este valor tiende el retardo al aumentar los usuarios, debido a que el incremento lo hacemos directamente en el DSLAM.

Canales Adyacentes

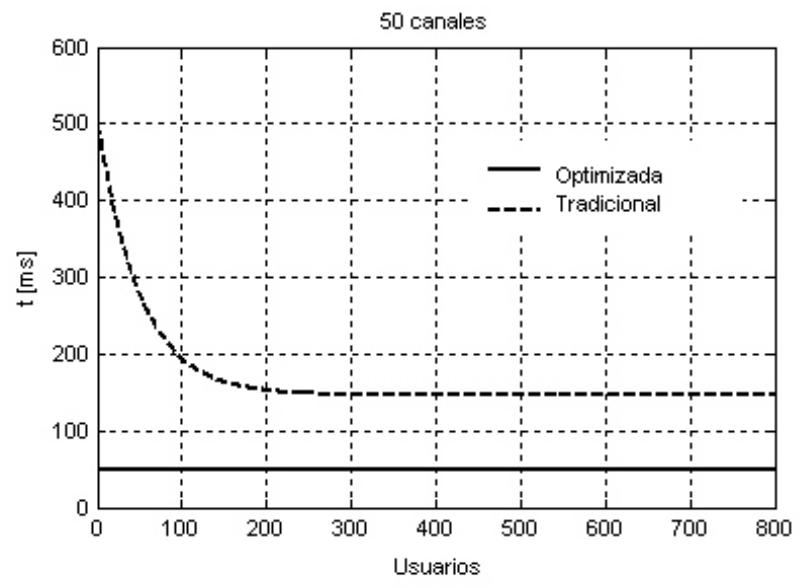

a)

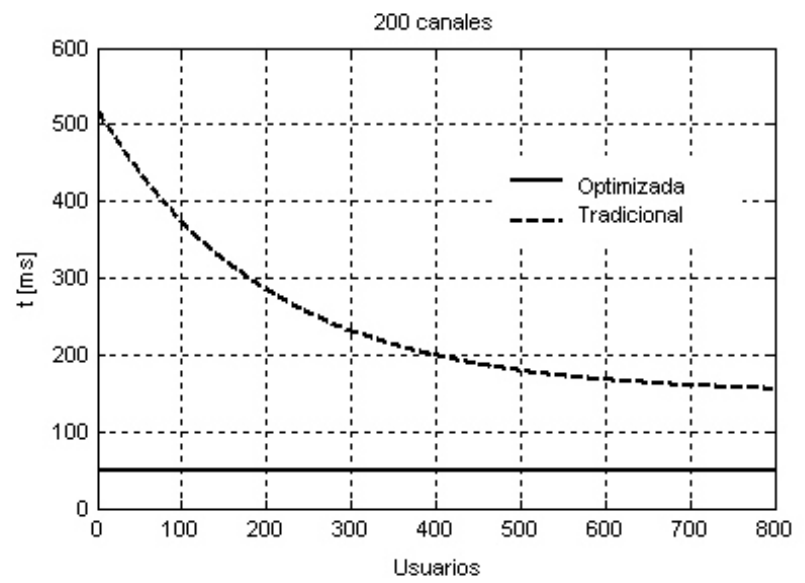

c)

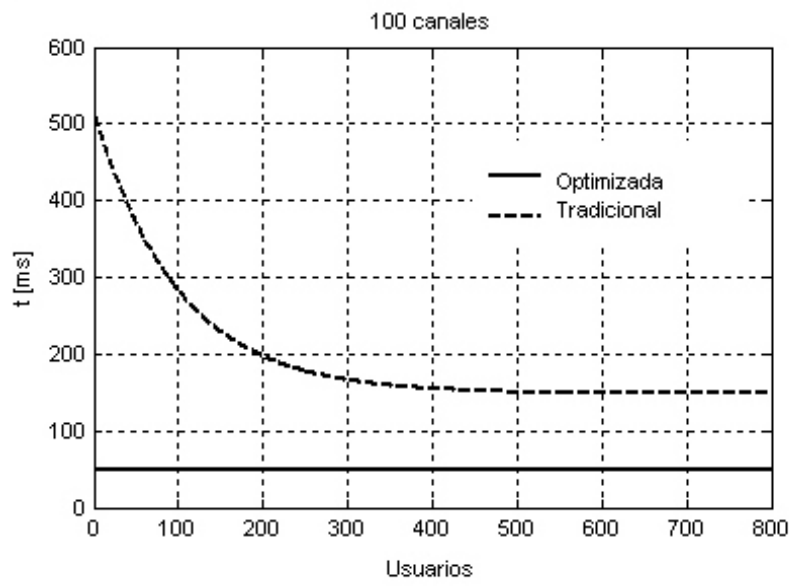

b)

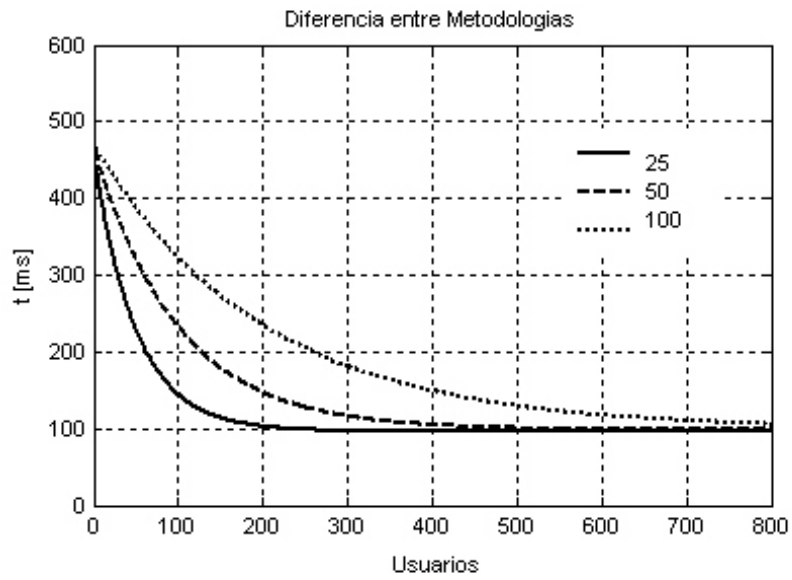

d)

Figura 8. Resultados obtenidos al variar el número de usuarios para cambios a canales adyacentes: a) 50 canales, b) 100 canales, c) 200 canales, d) Diferencia entre ambas tecnologías 
La figura 9 muestra las curvas para 50, 100 y 200 canales, haciendo un barrido del número de usuarios ahora para los escenarios 3 y 4 . La diferencia entre los resultados obtenidos por ambas metodologías también se ilustra. Cuando los usuarios cambian a canales aleatorios el retardo disminuye al aumentar el número de usuarios. Cuando el mé- todo de reducción es aplicado se tiene una pequeña reducción del tiempo de adquisición. Se observa nuevamente que el máximo beneficio del método de grupos adyacentes, cuyo valor es cercano a $150 \mathrm{~ms}$, se obtiene cuando el número de usuarios es la mitad al número de canales, debido a los hechos explicados anteriormente.

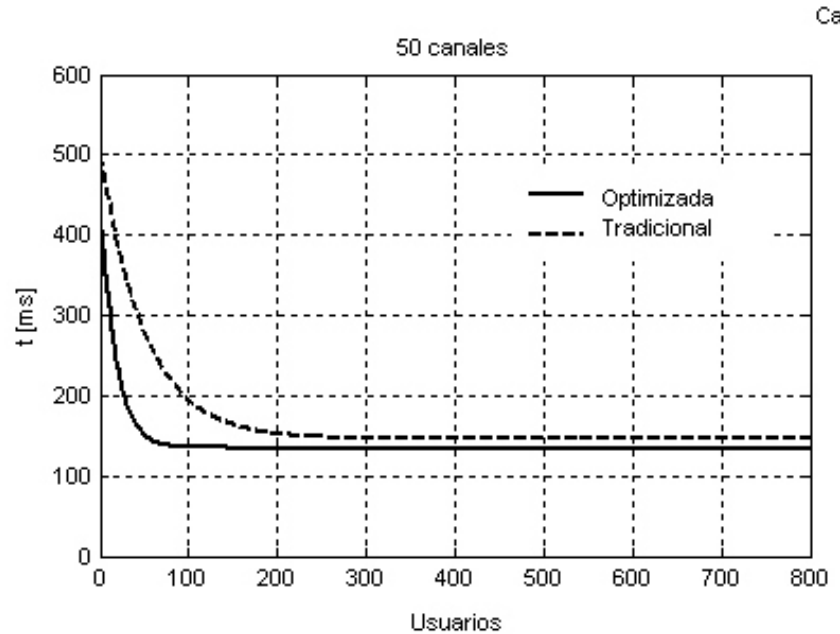

a)

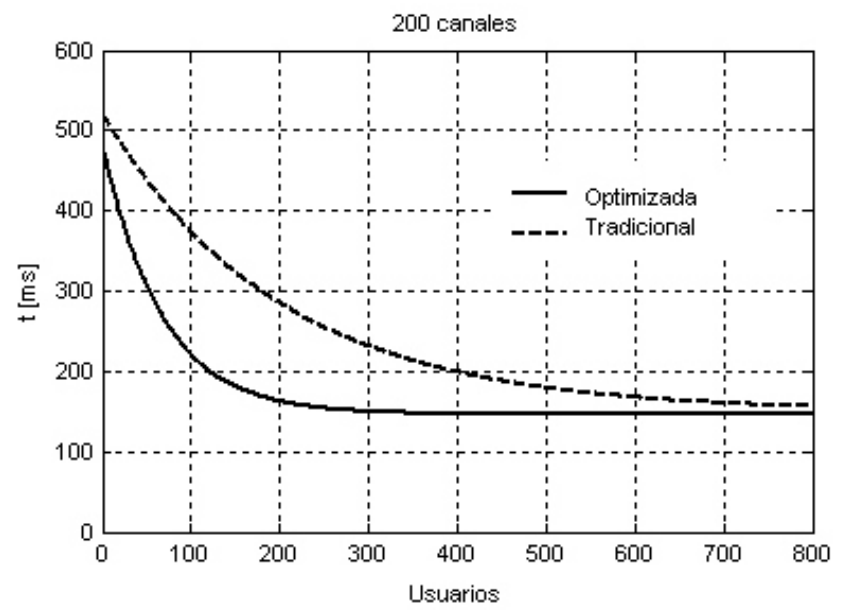

c)
Canales Aleatorios

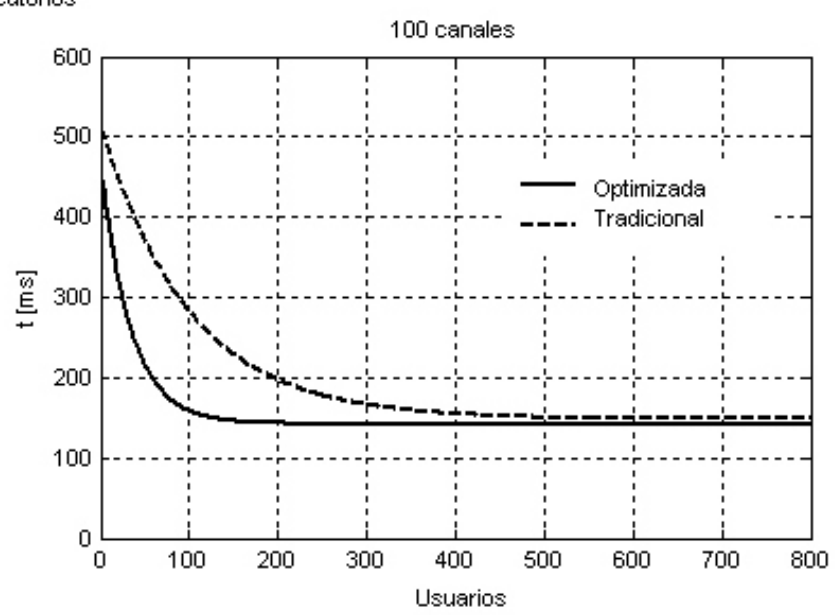

b)

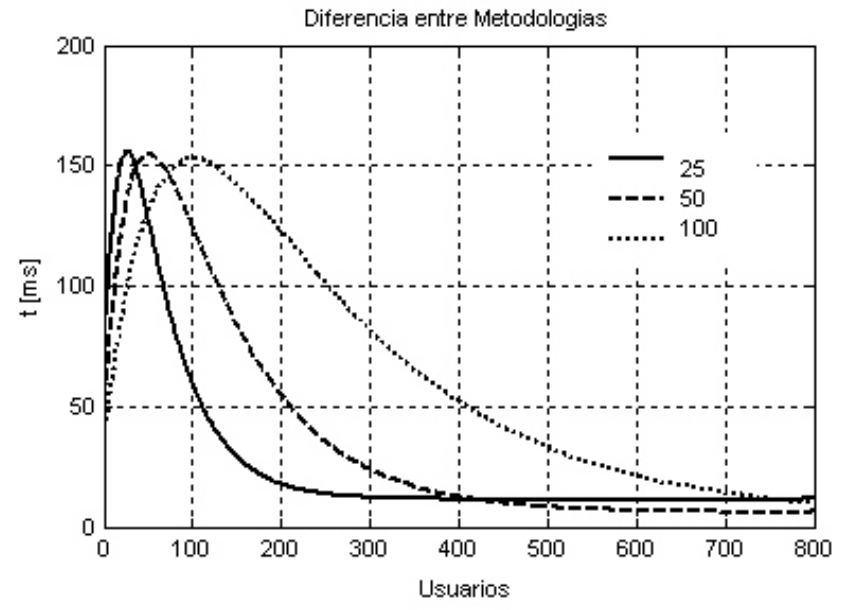

d)

Figura 9. Resultados obtenidos al variar el número de usuarios para cambios a canales aleatorios: a) 50 canales, b) 100 canales, c) 200 canales, d) Diferencia entre ambas tecnologías 
Finalmente, la figura 10 muestra un comparativo de los cuatro escenarios, primero para una variación de canales y 50 usuarios, después para 100 canales variando los usuarios.

Se puede apreciar en ambos casos que con el método tradicional, cambiando a canales adyacentes como a aleatorios, se tiene el mismo retardo, ya que el método tradicional trata igual a los canales adyacentes como aleatorios.

\section{Conclusiones}

Al implementar el método de grupos adyacentes se obtiene una mejora considerable del retardo si se cambia a canales adyacentes; el retardo tendrá un valor constante igual al tiempo entre la transmisión del Join message y la recepción del primer paquete del grupo multicast, correspondiente al nuevo canal enviado directamente del HG, por lo que el tiempo de adquisición será casi inmediato sin importar el número de canales o el de usuarios.

En el caso de cambios a canales aleatorios, al implementar el método de reducción, se logra una reducción del tiempo de adquisición que, si bien no es constante, se consigue la máxima reducción de este tiempo cuando el número de canales es igual al doble del número de usuarios; fuera de estos valores el método no ayudaría mucho en la reduc- ción del tiempo de cambio a canales aleatorios.

El método sólo servirá si el ancho de banda de la red de acceso es suficientemente grande para soportar diversos flujos multicast, de lo contrario, simplemente el método afectaría a la calidad de experiencia del usuario en vez de beneficiarla.

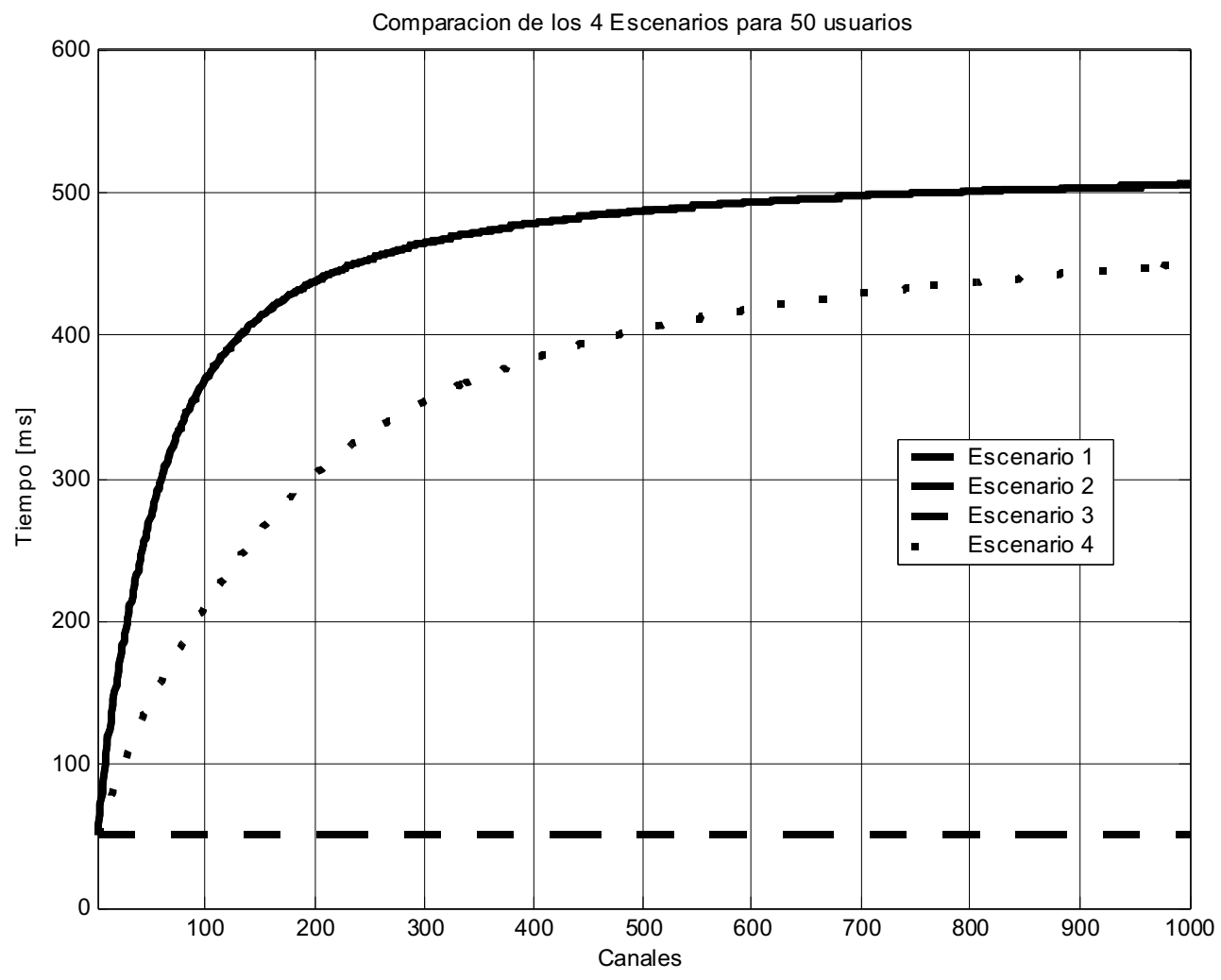

Figura 10. Gráficas comparativas de los cuatro escenarios (continúa...) 


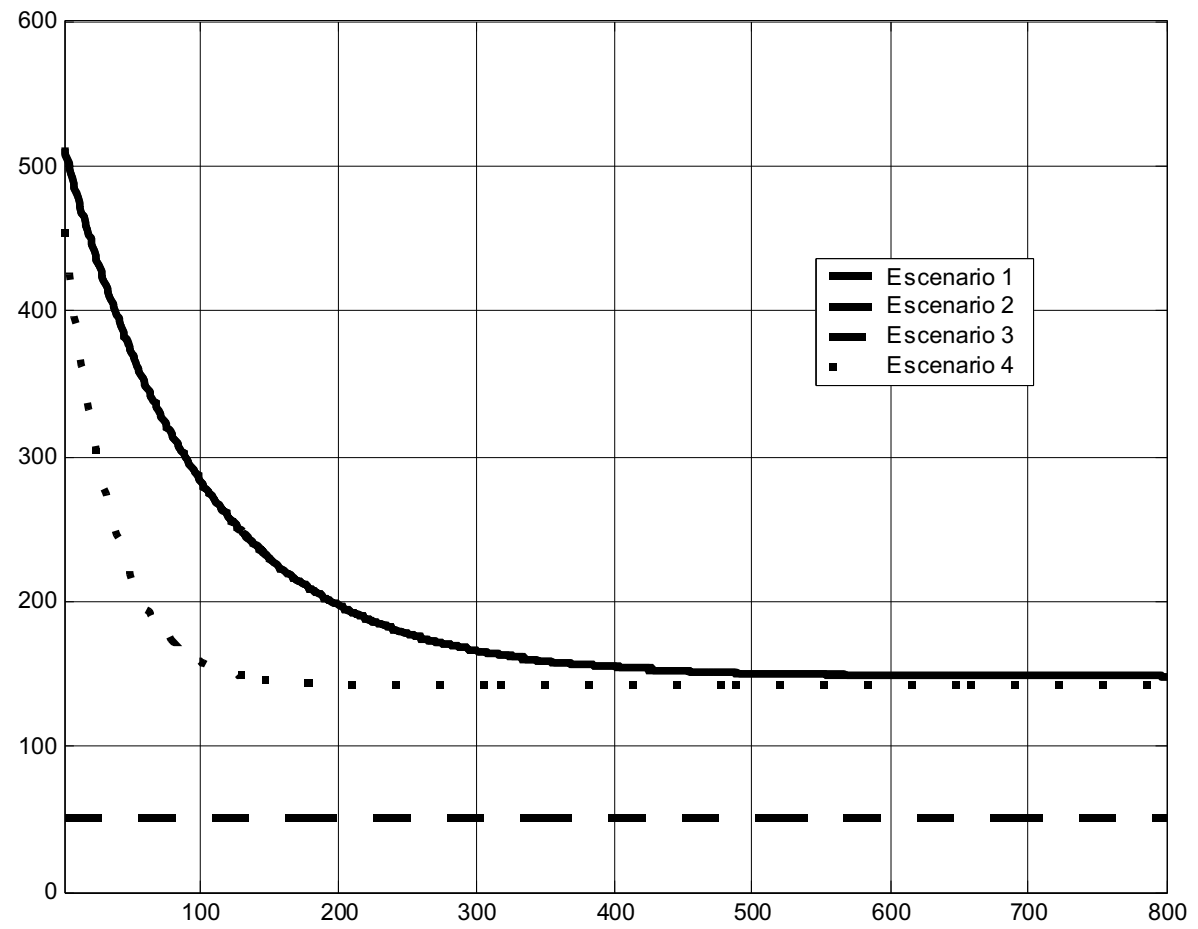

Figura 10. Gráficas comparativas de los cuatro escenarios (...continuación)

El método de grupos adyacentes es un buen método para reducir el tiempo de adquisición, que involucra una parte del tiempo de cambio de canal. No obstante, reducir los retardos de la red no es suficiente, sobre todo porque el mayor porcentaje del tiempo de cambio de canal concierne al proceso de decodificación y al diseño e implementación del STB. Estos aspectos merecen consideraciones especiales y probablemente, mejores diseños.

Reducir el retardo en el cambio de canal trae consigo un beneficio directo en la calidad de experiencia del usuario, permitiendo a los proveedores la captación de más suscriptores al sistema y provocando el abaratamiento de las tarifas del servicio.

Adicionalmente, se propone como trabajos a futuro, el análisis estadístico para la obtención del ancho de banda utilizado por la red, de acuerdo a la popularidad de cada canal, al número total de canales y de suscriptores. También se propone la obtención de los tiempos reales del retardo mediante la simulación del sistema en un software de redes o con una maqueta del modelo para recabar los tiempos de acuerdo a la utilización del sistema y a la distancia entre los ruteadores multicast.

\section{Referencias}

Chunglae C. et al. Improvement of channel zapping time in IPTV services using the adjacent groups Join-Leave method. In: 6th International Conference on Advanced Communication Technology, 2004, Vol. II, pp. 971-975.

Cisco Systems (2006a). Managing delay in IP video networks, USA. Version 1.0.

Cisco Systems. (2006b). Cisco wireline video/IPTV solution design and implementation Guide, Release 1.1, USA, pp. 376. 
DOI: http://dx.doi.org/10.22201/fi.25940732e.2008.09n3.017

F. Moumtadi, M. Escobar-Argota, R. López-Moreno y S. Landeros-ayala

Full Service-VDSL. FS-VDSL Specification, part 2, System Architecture Junio 2002. Full ServiceVDSL Committee.

Fenner B. et al. RFC 2362: Protocol independent multicast-Sparse mode (PIM SM): Protocol specification. Internet Engineering Task Force, Oct 2003.

Fenner W. RFC 2236: Internet Group Management Protocol, Version 2. Internet Engineering Task Force, Nov 1997.
Muñiz I. Televisión IP: Una experiencia totalmente personalizada [en línea]. Centro de Investigación e Innovación en Telecomunicaciones, A.C. [Fecha de consulta Julio 2005]. Disponible en: http://www.cinit.org.mx/articulo.php?idArticulo $=34$

Siemens Communications and Juniper Networks. High Quality and Resilient IPTV Multicast Architecture. Siemens Communications and Juniper Networks. 2006.

\section{Semblanza de los autores}

Fatima Moumtadi. Obtuvo su maestría en sistemas de radiodifusión satelital y su doctorado en televisión en la Facultad de Radiodifusión y Televisión de la Universidad Técnica de Comunicaciones e Informática de Moscú, Rusia (MTUCI). Se desarrolló profesionalmente en el área de Radiofrecuencia. Ha publicado artículos en congresos y revistas nacionales e internacionales. Actualmente es profesora de carrera en el Departamento de Telecomunicaciones en la Facultad de Ingeniería de la Universidad Nacional Autónoma de México.

Marlene Escobar-Argota. Obtuvo su título de ingeniera de telecomunicaciones en la Facultad de Ingeniería de la Universidad Nacional Autónoma de México. Actualmente trabaja en la empresa "Syspro Internacional".

Ricardo López-Moreno. Se título como ingeniero en telecomunicaciones en la Facultad de Ingeniería de la Universidad Nacional Autónoma de México. A la fecha labora en una empresa que posee y opera redes metropolitanas de fibra óptica llamada METRORED.

Salvador Landeros-Ayala. Egresó de la Facultad de Ingeniería, UNAM, con el título de ingeniero mecánico electricista en el área de comunicaciones. Cursó la maestría en ciencias de la ingeniería de telecomunicaciones en la Universidad Pennsylvania, Estados Unidos. Posteriormente, obtuvo el grado de doctor en ingeniería eléctrica en la Facultad de Ingeniería, UNAM. Ha escrito artículos que han sido presentados en congresos y revistas, tanto internacionales como nacionales. Fue miembro del Comité de Becas de CONACYT, director del Sistema de Satélites Nacionales y jefe de la División de Ingeniería Eléctrica. Actualmente es jefe de la División de Estudios de Posgrado de la Facultad de Ingeniería, UNAM. 